\title{
MODELO EXPERIMENTAL DE ESTEATOHEPATITE NÃO-ALCOÓLICA COM DIETA DEFICIENTE EM METIONINA E COLINA
}

\author{
Idilio ZAMIN Jr., Angelo Alves de MATTOS, Ângelo Zambam de MATTOS, \\ Eduardo MIGON, Ernesto SOARES e Marcos Luiz Santos PERRY
}

\begin{abstract}
RESUMO - Contexto - Ainda existem vários aspectos desconhecidos a respeito da esteatohepatite não-alcoólica, principalmente em relação à fisiopatologia e ao seu tratamento medicamentoso. Dessa forma, os modelos experimentais são importante para o melhor entendimento dessa doença, bem como para a avaliação do efeito das drogas. Objetivo - Desenvolver um modelo experimental de esteatohepatite não-alcoólica a partir do uso de dieta deficiente em metionina e colina. Métodos - Foram utilizados 50 ratos machos da linhagem Wistar. A dieta deficiente em metionina e colina foi processada de forma artesanal. Um grupo de 40 animais recebeu a dieta durante 90 dias e utilizou-se um grupo controle com 10 ratos que recebeu ração padronizada pelo mesmo período. Após, os animais foram mortos por decapitação e foi realizada laparotomia com hepatectomia total e preparo do material para análise macroscópica e histológica. O nível de significância foi $\alpha=0,05$. Resultados - Os ratos que receberam a dieta apresentaram perda significativa de peso, com achados de desnutrição e todos mostraram, pelo menos, algum grau de esteatose macrovesicular. O diagnóstico de esteatohepatite não-alcoólica foi realizado em 27 (70\%) dos 39 ratos que receberam a dieta. Nenhum dos 10 ratos que recebeu ração apresentou alterações histológicas. Conclusão - A dieta com restrição de metionina e colina desenvolvida apresenta índices elevados de indução de esteatose e esteatohepatite em modelo animal com baixo custo.
\end{abstract}

DESCRITORES - Fígado gorduroso. Dieta. Metionina, deficiência. Deficiência de colina. Ratos.

\section{INTRODUÇÃO}

A esteatohepatite não-alcóolica (EHNA) é caracterizada por acúmulo de gordura no fígado associado a graus variáveis de inflamação e fibrose. Esta doença apresenta forte associação com obesidade, sexo feminino e diabete melito (DM) $)^{(5,30,31,48)}$

Atualmente, no ocidente, a EHNA tem sido considerada ora a segunda ${ }^{(12)}$, ora a terceira ${ }^{(9)}$ doença hepática mais comum em pacientes de ambulatório. Em nosso meio, ao serem avaliados prospectivamente, 912 indivíduos obesos, sem DM associado, em ambulatório de nutrição, foi possível determinar, pela primeira vez em estudo populacional e não de uma série de casos, prevalência de esteatohepatite não-alcoólica da ordem de $3,18 \%{ }^{(56)}$. Essa prevalência, no entanto, é subestimada, uma vez que só foram avaliados os pacientes com alterações de aminotransferases.

A despeito de apresentar alta prevalência na população, a história natural de EHNA ainda é pouco estudada. Atualmente, acredita-se que a EHNA possa evoluir para cirrose e carcinoma hepatocelular $(\mathrm{CHC})$, enquanto a esteatose isolada não costuma apresentar esta evolução. Ressalta-se existirem poucos estudos prospectivos na literatura, com biopsias sequenciais, em pacientes com EHNA com o intuito de avaliar a progressão da doença ${ }^{(5,25,43)}$.

O reconhecimento do seu potencial evolutivo e a sua alta prevalência são os principais motivos que despertam o interesse da comunidade científica em relação ao seu estudo $^{(4,5,8,9,13,25,30,32,43,44,48,49,50,51,53,55)}$

A respeito do tratamento da EHNA, até o presente momento, nenhuma terapia específica mostrou resultados conclusivos. Enquanto a maioria dos autores acredita que os pacientes que apresentam esteatose devem ser apenas observados e avaliados periodicamente, muito tem se estudado em relação à terapêutica da EHNA. Embora alguns trabalhos sugiram que a redução gradual de peso em pacientes obesos ${ }^{(14,38,43)}$ e a realização regular de atividade física aeróbica possam ter efeito benéfico ${ }^{(2,4,12,35,46,55)}$, mais estudos com seguimento histológico são necessários para demonstrar se essa conduta ocasiona regressão das lesões hepáticas a longo $\operatorname{prazo}^{(3,11,52)}$.

Em relação ao tratamento medicamentoso da EHNA, há poucos estudos testando drogas em seres

Curso de Pós-Graduação em Hepatologia da Fundação Faculdade Federal de Ciências Médicas de Porto Alegre, RS.

Correspondência: Dr. Idílio Zamin Jr. - Rua Armando Pereira Câmara, 111 - apt. 801 - 90470-070 - Porto Alegre, RS. E-mail: izamin@terra.com.br 
humanos ${ }^{(10,16,21,22,23,29,31,33,36,54)}$, sendo que uma das grandes dificuldades observadas é a necessidade da realização de segunda biopsia hepática, uma vez ser esta a única maneira fidedigna de avaliar o efeito da droga na evolução da EHNA. Assim, torna-se de grande relevância testar a eficácia terapêutica em modelos animais de EHNA.

Tendo em vista o custo de linhagens de ratos geneticamente modificadas para a realização destes estudos, é importante que se consiga um modelo experimental de EHNA adaptado à necessidade do nosso meio.

Este estudo teve como objetivo desenvolver um modelo experimental de EHNA a partir do uso de dieta deficiente em metionina e colina (DMC).

\section{MÉTODO}

\section{Animais}

Nesse experimento, foram utilizados 50 ratos machos, com 45 dias de idade, pesando entre 200 e 250 g, da espécie Rattus norvegicus, da linhagem Wistar, heterozigotos. Os animais foram provenientes do Biotério da Fundação Faculdade Federal de Ciência Médicas de Porto Alegre (FFFCMPA), Porto Alegre, RS. Esses ratos foram mantidos em gaiolas de polipropileno com dimensões de $47 \times 34$ x $18 \mathrm{~cm}$, em conjuntos de cinco animais por gaiola, sob um ciclo claro/escuro de $12 \mathrm{~h}$ (ciclo claro das 7 às $19 \mathrm{~h}$ ) e sob condições controladas de temperatura $\left(22^{\circ} \mathrm{C} \pm 2,0^{\circ} \mathrm{C}\right)$.

\section{Dieta}

A dieta DMC foi processada no Laboratório da Faculdade de Bioquímica da Universidade Federal do Rio Grande do Sul, segundo o modelo de ROGERS e NEWBERNE ${ }^{(45)}$ modificado, para a indução de EHNA, sendo composta por:

- caseína livre de vitaminas 6\%;

- óleo de soja $45 \%$;

- amido $41 \%$;

- fibras $1 \%$;

- mistura vitamínica $2 \%$ (acetato de vitamina A 0,03; vitamina D 0,003 ; menadiona 0,001 ; acetato de vitamina E 0,045 ; tiamina 0,001 ; riboflavina 0,002 ; piridoxina 0,001 ; ácido p-aminobenzóico 0,01 ; biotina 0,00005 ; nicotinamida 0,005 ; pantotenato de cálcio 0,005; inositol 0,02; ácido ascórbico $0,02)$

- sais $5 \%$.

\section{Procedimento experimental}

$O$ modelo de indução de EHNA foi realizado mediante alimentação dos animais com dieta pobre em metionina e colina $^{(45)}$ ad libitum durante 90 dias. A água foi oferecida ad libitum através de mamadeiras de vidro com bico de inox em rolha de borracha.

$\mathrm{O}$ animais foram divididos, aleatoriamente, em dois grupos:

- grupo 1: 10 ratos receberam ração padronizada (Nuvilab CR-1- Nuvital Nutrientes Ltda ${ }^{\circledR}$ ) durante 3 meses;

- grupo 2: 40 ratos receberam dieta deficiente em metionina e colina durante 3 meses.

\section{Coleta dos materiais e morte dos animais}

No primeiro dia do estudo, foi determinado o peso dos animais. Um dia após o término do período de indução (90 dias), os animais foram novamente pesados e mortos por decapitação. Então, foi realizada laparotomia com hepatectomia total e preparo do material para análise macroscópica e histológica.

Os fígados foram seccionados e o lobo direito colocado em fixador de Bouin (solução composta por ácido pícrico, formol e ácido acético), permanecendo nessa solução por aproximadamente 4 horas, para, então, ser lavado em água corrente. Após, o material foi transferido para frascos com álcool $50 \%$, onde permaneceu por 30 minutos. Por último, os fígados foram armazenados em frascos com álcool $70 \%$, aguardando o processo para inclusão em parafina.

\section{Análise histológica}

Inicialmente, foi realizado estudo macroscópico dos fígados retirados. Para a avaliação microscópica, as lâminas dos fragmentos hepáticos foram coradas com hematoxilina-eosina, picro-sirius, para avaliação do grau de fibrose, e Perls, para avaliação da presença de depósitos de ferro, tendo sido examinadas por um único patologista, "às cegas" em relação aos dados dos animais.

O critério histológico mínimo para o diagnóstico de EHNA foi a presença de esteatose associada à balonização hepatocelular, envolvendo a zona 3 e infiltrado inflamatório $\operatorname{lobular}^{(7,17)}$. Os corpúsculos de Mallory e a fibrose sinusoidal, envolvendo a zona 3, podiam ou não estar presentes ${ }^{(7,17)}$.

A graduação tanto da atividade necroinflamatória como da fibrose foi realizada, segundo a classificação proposta por BRUNT et al. ${ }^{(7)}$

\section{Comitê de Ética}

Este projeto de pesquisa foi submetido e aprovado pelo Comitê de Ética em estudo animal da FFFCMPA. Todos os ratos foram manipulados, observando-se rigorosamente, as normas institucionais para trabalhos experimentais com animais preconizadas por esse Comitê.

\section{Análise estatística}

Foram calculadas as médias e o desvio padrão de todas as variáveis quantitativas e ordinais. No caso dos escores, optouse por realizar transformação em postos antes da comparação dos grupos. A comparação foi feita por análise de variância com critério de classificação, que quando aplicada sobre os postos, é equivalente ao procedimento de Kruskal-Wallis. A localização de eventuais diferenças entre os grupos baseou-se no procedimento de Tukey, executado sobre os postos (quando necessário). As variáveis dicotômicas foram comparadas pelo teste exato de Fisher. O nível de significância do estudo foi estabelecido em $\alpha=0,05$. Os dados foram analisados no programa SPSS v12.0 $0^{(1,34)}$

\section{RESULTADOS}

Dos 50 ratos que foram submetidos ao estudo, 49 completaram os 90 dias da dieta. Apenas um rato foi a óbito durante o período de estudo. 


\section{Peso dos animais}

$\mathrm{O}$ peso inicial dos animais foi semelhante entre os grupos, porém os ratos que receberam a dieta DMC apresentaram perda significativa de peso ao final dos 90 dias, com achados de desnutrição. Ao contrário, o grupo que recebeu ração apresentou ganho de peso (Tabela 1 e Figuras 1 e 2).

TABELA 1. Peso dos ratos $(\mathrm{g})$ antes e após a dieta

\begin{tabular}{lccc}
\hline Grupo & Peso inicial $(\mathrm{g})$ & Peso final $(\mathrm{g})$ & $\boldsymbol{P}$ \\
\hline Ração & $260,7 \pm 24,9$ & $368,1 \pm 32,9$ & $<0,001$ \\
Dieta & $270,7 \pm 18,6$ & $226,1 \pm 18,3$ & $<0,001$ \\
\hline
\end{tabular}

Os dados são apresentados como média \pm desvio padrão

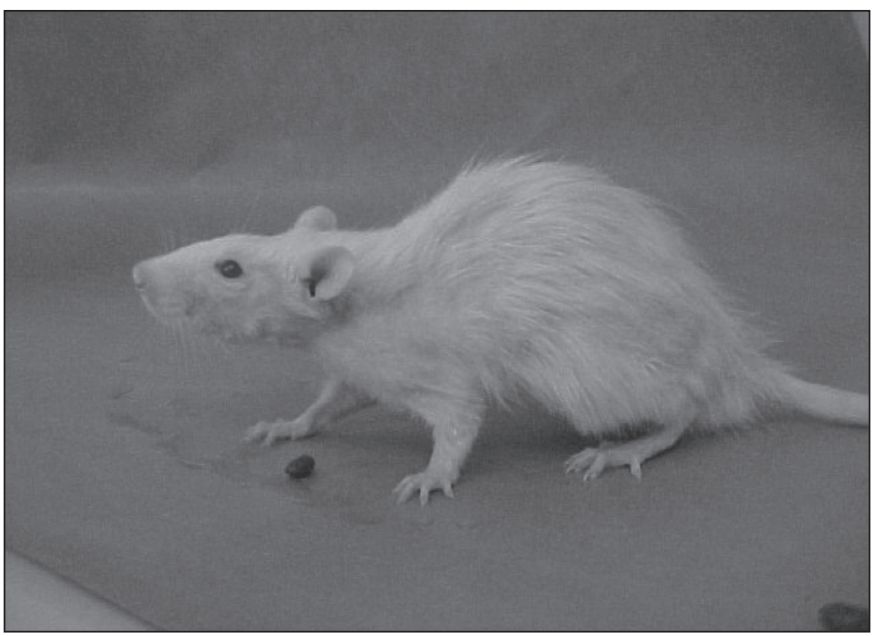

FIGURA 1. Animal que recebeu a dieta DMC após 90 dias

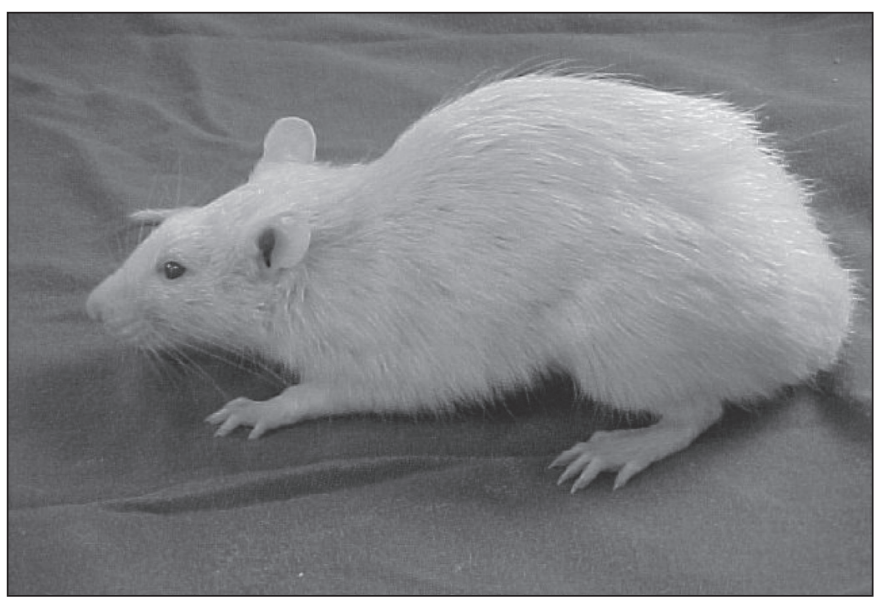

FIGURA 2. Animal que recebeu ração normal para ratos durante 90 dias

\section{Aspecto macroscópico dos fígados}

Em relação ao exame macroscópico dos fígados, todos os animais que receberam ração apresentaram fígado com aspecto e coloração normal. Enquanto isso, os animais que receberam a dieta DMC apresentaram fígado aumentado de tamanho, com coloração esbranquiçada e, em alguns espécimes, puderam ser observadas áreas amareladas de depósitos de gordura (Figuras 3 e 4).

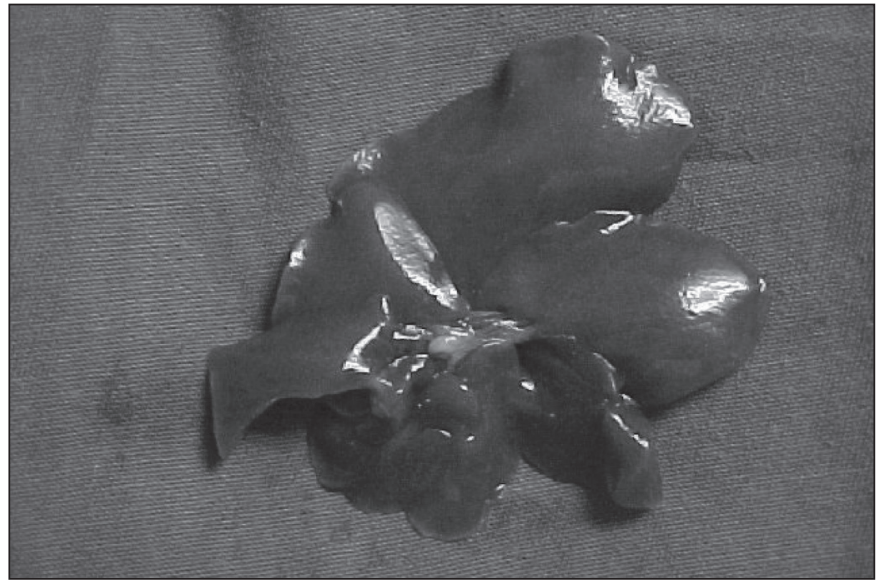

FIGURA 3. Aspecto macroscópico de um fígado do grupo que recebeu ração

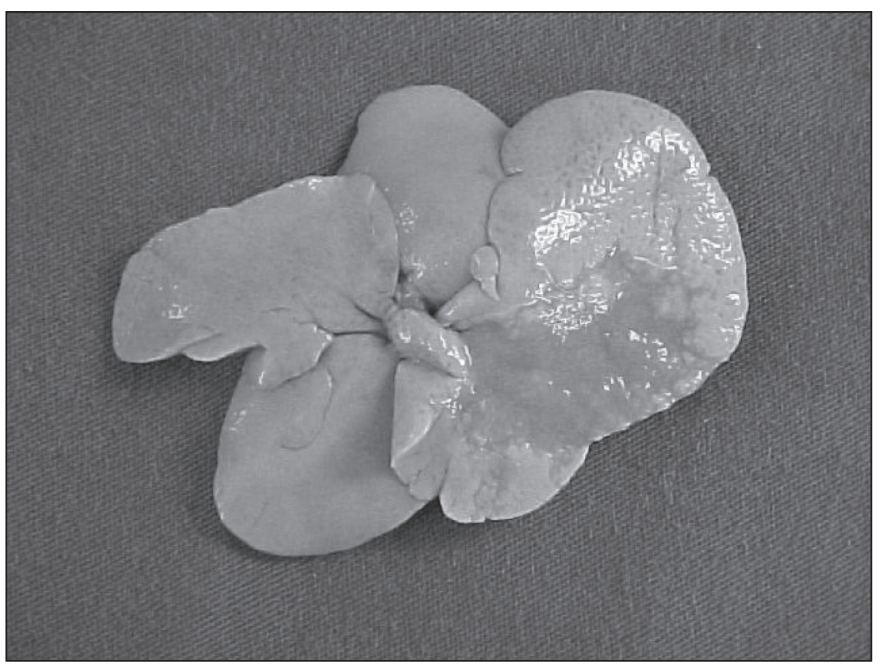

FIGURA 4. Aspecto macroscópico de um fígado do grupo que recebeu a dieta DMC

\section{Histologia}

Em relação aos achados histológicos, nenhum dos 10 ratos que recebeu ração apresentou alterações histológicas, sendo considerados como tendo fígado normal.

Dentre os 39 ratos que receberam a dieta DMC, todos apresentaram, pelo menos, algum grau de esteatose macrovesicular, que sempre foi predominante em relação à microvesicular.

O diagnóstico de esteatohepatite não-alcoólica foi realizado em $27(70 \%)$ dos 39 ratos que receberam a dieta DMC (Figura 5).

Em relação à atividade inflamatória, esta esteve ausente em 12 ratos, sendo que 13 ratos apresentaram grau 1,8 grau 2 , e outros 6 animais apresentaram grau 3.

Embora ausente em 19 casos, a fibrose foi considerada estágio $1 \mathrm{em} 13$ ratos, estágio $2 \mathrm{em} 1$, estágio $3 \mathrm{em} \mathrm{5,} \mathrm{e} \mathrm{um} \mathrm{rato}$ apresentou cirrose (estágio 4). Em relação aos demais aspectos histológicos, não foram observados depósitos de ferro e a presença de corpúsculos de Mallory ocorreu em apenas cinco casos. 


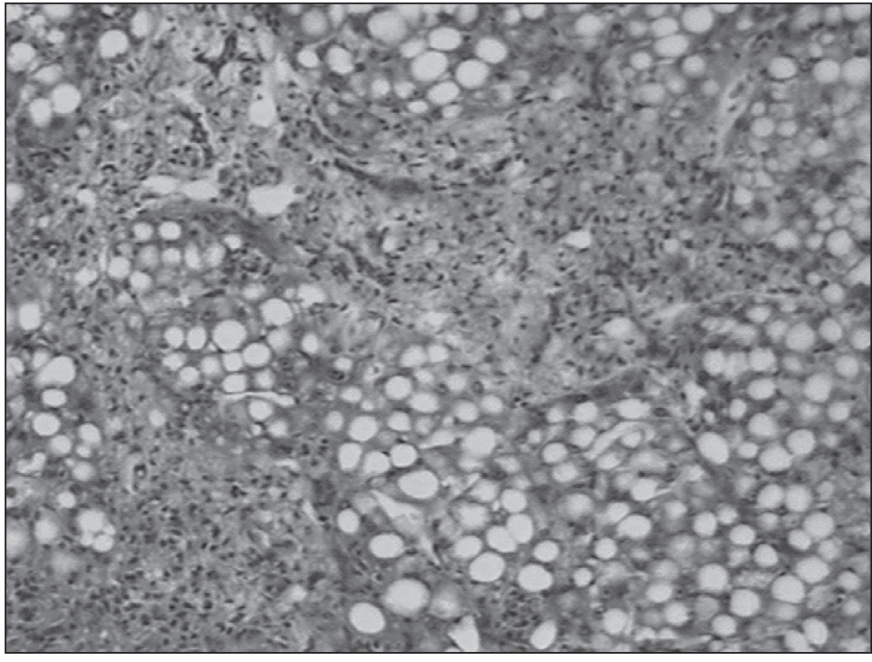

FIGURA 5. Fotomicrografia demonstrando esteatose macrovesicular acentuada e atividade necroinflamatória (H-E, 200X)

\section{DISCUSSÃO}

A utilização de modelos animais com EHNA é muito importante no estudo desta doença, pois pode permitir melhor entendimento de sua fisiopatologia, ajudar a esclarecer os mecanismos envolvidos na transição da esteatose para a EHNA, bem como testar o resultado das várias drogas disponíveis para o seu tratamento. A indução de EHNA tem sido realizada de diferentes maneiras: por indução medicamentosa (tetraciclina, amiodarona, corticosteróides, entre outros); com a utilização de ratos geneticamente obesos ou com manipulação genética e com a utilização de dietas que promovem a sua ocorrência, seja por serem ricas em gordura, seja por restrição de aminoácidos ${ }^{(19,26,40,45,54)}$.

As drogas podem induzir esteatose e EHNA ao inibir a $\beta$-oxidação mitocondrial de ácidos graxos, como ocorre com a amiodarona, as tetraciclinas e os corticosteróides, entre outros, ou também, ao bloquear a liberação de triacilgliceróis do fígado, proporcionando consequentemente, o seu acúmulo, como ocorre, por exemplo, com o ácido valpróico ${ }^{(26)}$. LETTÉRON et al. ${ }^{(26)}$ conseguiram a indução de esteatose em ratos com a administração de diversas drogas (amiodarona, doxiciclina, etanol, tetraciclina, ácido valpróico e dexametasona) em diferentes raças de animais. A maioria dos animais apresentou esteatose predominantemente microvesicular, à exceção do grupo que recebeu etanol, no qual a forma de esteatose predominante foi macrovesicular. Este modelo de indução de esteatose e esteatohepatite, apesar de eficaz, certamente, na maioria das vezes, não traduz patogênese semelhante à da EHNA e também pode refletir a hepatotoxicidade específica da droga, confundindo os resultados dos estudos quando avaliado o papel de determinado medicamento em sua prevenção. Desta forma, não parece ser a melhor opção para modelos experimentais que se propõem a estudar a EHNA.

Os modelos genéticos de EHNA utilizam animais que possuem alguma mutação espontânea que promove a doença ou linhagens de animais geneticamente modificadas em laboratório, que desenvolverão EHNA ${ }^{(20)}$.
Os ratos ob/ob são o principal exemplo de animais que apresentam mutação espontânea que bloqueia a produção de leptina ${ }^{(20,39)}$. À semelhança dos humanos, estes animais apresentam hiperinsulinemia (resistência à insulina), hiperglicemia e dislipidemia, e desenvolvem esteatose ${ }^{(39)}$. Na deficiência de leptina, ocorre importante esteatose, porém a esteatohepatite somente ocorre se houver outro estímulo hepatotóxico como, por exemplo, o uso de alguma droga ou a indução de isquemia ${ }^{(47)}$.

Outra linhagem de ratos geneticamente obesos são os fa/fa, que desenvolvem mutação espontânea no gene do receptor da leptina ${ }^{(41)}$. Desta forma, apesar de produzirem o hormônio, apresentam resistência periférica ao mesmo, com efeito final semelhante ao que ocorre com os ratos ob/ob, porém sem responder à administração exógena de leptina ${ }^{(20)}$.

Em laboratório de genética, consegue-se realizar modificações gênicas e a produção de linhagens de animais que desenvolvem esteatose. Existem várias intervenções que podem ser realizadas em genes diferentes com objetivo semelhante, ou seja, gerar desequilíbrio na homeostasia dos lipídios, de forma que ocorra a formação de esteatose. Uma das principais modificações genéticas utilizadas nesses modelos é a ativação de genes envolvidos na síntese de ácidos graxos e colesterol, promovendo a lipogênese e a deposição de gordura no fígado ${ }^{(20)}$.

Tanto os ratos com mutação natural que induz a esteatose e a EHNA, como aqueles geneticamente modificados, são excelentes modelos experimentais para estudo desta doença e são utilizados, rotineiramente, com tal propósito em pesquisas ${ }^{(6,18,28)}$. Tratam-se, porém, de modelos onerosos, pela dificuldade de aquisição de linhagens com mutação espontânea ou pela necessidade de técnicas sofisticadas realizadas em laboratório de biologia molecular, pouco disponíveis em nosso meio. Dessa forma, torna-se difícil a utilização desses modelos na maioria das pesquisas.

A indução de EHNA também é possível em modelos animais através de manipulação da dieta, de forma a promover a deposição de lipídios no fígado. Nesse sentido, podem-se utilizar dietas que promovam a obesidade e a esteatose ou dietas que induzam somente a deposição de gordura hepática, sem a ocorrência de obesidade.

Como a doença hepática gordurosa não-alcoólica (DHGNA) está fortemente associada à obesidade, alguns estudos em modelos experimentais a induzem e, dessa forma, esperam a ocorrência posterior de esteatose e EHNA. Entretanto, à semelhança dos seres humanos, os ratos apresentam respostas e suscetibilidade diferentes à dieta, sendo que tanto a ocorrência de obesidade como posteriormente a DHGNA, dependem de outros fatores, como idade, gênero e predisposição genética. Dentre as diversas dietas com este propósito, aquelas com elevadas quantidades de lipídios ou glicídios são as mais utilizadas ${ }^{(27,42)}$.

Ao contrário do que acontece com as dietas anteriormente descritas, os animais que são alimentados com dieta deficiente em metionina e colina apresentam perda de peso e importante desnutrição ${ }^{(19,54)}$. Esses aminoácidos essenciais possuem papel fundamental na síntese das apoproteínas, que são proteínas especializadas no transporte dos lipídios no plasma, favorecendo, na sua ausência, o acúmulo dos mesmos no fígado ${ }^{(19,54)}$. Além disso, com a dieta DMC, a ß-oxidação mitocondrial de lipídios fica reduzida, contribuindo para a ocorrência de esteatose. O mecanismo da produção desse modelo também se vale do fato 
de que a metionina e a colina são precursores essenciais para a síntese hepática de fosfatidilcolina que, junto com o colesterol, perfaz a maioria absoluta dos lipídios secretados na bile ${ }^{(20)}$.

Historicamente, essa dieta foi descrita por ROGERS e NEWBERNE ${ }^{(45)}$, em 1973, ao observarem que poderia ocorrer o desenvolvimento de cirrose em um modelo animal, apenas com manipulação da dieta e sem o uso de álcool. Os autores demonstraram que a utilização de dieta deficiente dos aminoácidos colina e metionina, mas rica em lipídios, induz importante esteatose e doença hepática semelhante à observada com o uso de álcool. Quanto mais deficiente em aminoácidos, ficou comprovada maior velocidade de instalação e gravidade da doença hepática. Nesse estudo, os autores descreveram e testaram três composições com graus progressivos de deficiência nutricional, mas não relataram a percentagem de esteatose e EHNA obtida com cada dieta. Esse modelo de dieta DMC vem sendo utilizado até hoje com o propósito de indução de EHNA em modelos animais.

No presente estudo, foram encontradas várias dificuldades para a elaboração da dieta e, em modelo inicial, ocorreu falha na indução da esteatose e EHNA, apesar de ter sido seguida a base da fórmula descrita originalmente. Somente após adaptação da dieta, de acordo com as substâncias disponíveis em nosso meio, é que foi conseguido êxito na indução da doença. Entre outros fatores, foi observado que os ratos têm a capacidade de sintetizar a metionina e a colina por rota metabólica alternativa, a partir do ácido fólico, que também deve ser removido da dieta para que a mesma seja eficaz.

OKAN et al. ${ }^{(37)}$, com a finalidade de testar o papel do ácido ursodesoxicólico na EHNA, realizaram sua indução em ratos Wistar, utilizando dieta DMC. Os autores empregaram uma forma industrializada da dieta, disponível comercialmente nos Estados Unidos. Ao final de 30 dias, foi observada esteatose em $19(52,7 \%)$ dos 36 ratos submetidos a dieta. A EHNA, porém, ocorreu em apenas cinco $(13,8 \%)$ ratos. Por outro lado, WELTMAN et al. ${ }^{(54)}$, também utilizando uma forma comercial da dieta DMC, conseguiram a indução de esteatose em todos os seis animais submetidos ao estudo, após 4 semanas de tratamento. Como o objetivo do estudo era avaliar a atividade do citocromo CYP2E1 e a lipoperoxidação, os autores não citaram quantos ratos desenvolveram EHNA. Chamaram atenção, entretanto, para a importante perda de peso que esta dieta induz nos animais.

A maioria dos estudos em modelos animais ${ }^{(24,37,54)}$ que utiliza a dieta DMC para a indução de EHNA, usou uma forma industrializada dessa dieta e somente citou o laboratório que a produziu. Apenas GRATTAGLIANO et al. ${ }^{(15)}$, apesar de também utilizarem forma industrial da dieta, descreveram detalhadamente sua fórmula. Nesse estudo, após 10 dias de dieta, os autores observaram esteatose macrovesicular significativa em todos os 14 animais avaliados porém, provavelmente pelo curto período, não foi observada a ocorrência de EHNA.

No presente estudo, foi utilizada a dieta DMC para a indução de EHNA, desenvolvida de maneira artesanal, seguindo o modelo proposto por ROGERS e NEWBERNE ${ }^{(45)}$. Todos os animais estudados, com exceção de um, concluíram o protocolo proposto.

À semelhança dos outros estudos ${ }^{(15,24,37,54)}$, os animais que utilizaram a dieta DMC apresentaram perda significativa de peso ao final dos 90 dias de tratamento, com sinais inequívocos de desnutrição protéico-calórica.

Com a administração dessa dieta por 90 dias, obteve-se a indução de esteatose em 100\% dos animais. Esta pôde ser avaliada já macroscopicamente, pois os animais submetidos ao tratamento apresentaram fígado aumentado de tamanho, com coloração esbranquiçada, podendo ser observadas áreas amareladas de depósitos de gordura em alguns espécimes. Ao analisar-se a histologia, todos os ratos apresentaram, pelo menos, algum grau de esteatose macrovesicular, que sempre foi predominante em relação à microvesicular, indo ao encontro dos demais estudos que utilizam dieta DMC para a indução de $\operatorname{EHNA}^{(15,24,37,54)}$.

O diagnóstico de esteatohepatite não-alcoólica foi realizado em $27(70 \%)$ dos 39 animais submetidos a dieta DMC. Esses achados confirmam que a dieta utilizada é altamente eficaz para a indução da doença. Os estudos que utilizam a dieta DMC não especificam exatamente quantos animais desenvolveram a EHNA para comparação.

O presente estudo comprovou, pois, que a utilização de dieta com restrição de metionina e colina, produzida de forma não-comercial, é eficaz e se destaca, entre as várias maneiras de induzir EHNA em modelos animais, pelo baixo custo e pelo fato de poder ser facilmente manipulada em laboratório, sem a necessidade de técnicas sofisticadas. É também importante destacar o fato deste modelo não necessitar o uso de droga para a indução da doença e, principalmente, de apresentar elevados índices de indução de esteatose e EHNA. Assim, fica sugerido este modelo como padrão para os estudos experimentais em EHNA, uma vez que apresenta baixo custo e elevada eficácia.

Zamin Jr I, Mattos AA, Mattos AZ, Migon E, Soares E, Perry MLS. Model of experimental nonalcoholic steatohepatitis from use of methionine and choline deficient diet. Arq Gastroenterol. 2009;46(1): 69-74.

ABSTRACT - Context - There are still many unknown aspects about nonalcoholic steatohepatitis, especially regarding its pathophysiology and pharmacological treatment. Thus, experimental models are important for a better understanding of this disease and the evaluation of the effects of drugs. Objective - To develop a model of experimental nonalcoholic steatohepatitis from use of methionine and choline deficient diet. Methods - Fifty Wistar male rats were studied. A methionine and choline deficient diet has been processed in a craft. A group of 40 animals received the deficient diet for 90 days, and a group of 10 rats (control group) received the standardized ration in the same period. After, the animals were killed by decapitation, and laparotomy was performed. Hepatectomy was performed and the liver was studied by macroscopy and microscopy. The level of significance considered was of 0,05. Results - The rats that received the deficient diet showed significant loss of weight with findings from malnutrition and all of them had at least some degree of macrovesicular steatosis. The diagnosis of nonalcoholic steatohepatitis was performed in 27 (70\%) of the 39 rats that received this deficient diet ( 1 rat died during the study). None of the 10 rats that received the standardized diet had histological abnormalities. Conclusion - The diet restricted in methionine and choline induced steatosis and steatohepatitis in an animal model with low cost.

HEADINGS - Fatty liver. Diet. Methionine, deficiency. Choline deficiency. Rats. 


\section{REFERÊNCIAS}

1. Altman Dg. Practical statistics for medical research. London: Chapman \& Hall; 1991. $611 \mathrm{p}$

2. American Gastroenterological Association. American Gastroenterological Association medical position statement: nonalcoholic fatty liver disease. Gastroenterology. 2002;123:1702-4.

3. Anderson T, Gluud C, Franzmann Mb. Hepatic effects of dietary weight loss in morbidly obese subjects. J Hepatol. 1991;12:224-9.

4. Angulo P. Nonalcoholic fatty liver disease. N Engl J Med. 2002;346:1221-31.

5. Bacon Br, Farahvash MJ, Janney CG, Neuschwander-Tetri BA. Nonalcoholic steatohepatitis: an explanded clinical entity. Gastroenterology. 1994;107:1103-9.

6. Baffy G, Zhang CY, Glickman JN, Lowell BB. Obesity-related fatty liver is unchanged in mice deficient for mitochondrial uncoupling protein 2. Hepatology. 2002;35:753-61.

7. Brunt EM, Janney CG, Di Bisceglie AM, Neuschwander-Tetri BA, Bacon B Nonalcoholic steatohepatitis: a proposal for grading and staging the histological lesions. Am J Gastroenterol. 1999;94:2467-74.

8. Bugianesi E, Leone N, Vanni E, Marchesini G, Brunello F, Carucci P, Musso A, Paolis P, Capussotti L, Salizzoni M, Rizzetto M. Expanding the natural history of nonalcoholic steatohepatitis: from cryptogenic cirrhosis to hepatocellular carcinoma. Gastroenterology. 2002;123:134-40.

9. Caldwell Sh, Oelsner DH, Iezzoni JC. Cryptogenic cirrhosis: clinical characterization and risk factors for underlying disease. Hepatology. 1999;29:664-9.

10. Caldwell Sh, Hespenheide EE, Redick JA, Tezzoni JC, Battle EH, Sheppard BL. A pilot study of a thiazolidinedione, troglitazone, in nonalcoholic steatohepatitis. Am J Gastroenterol. 2001;96:519-25.

11. Capron Jp, Delamarre J, Dupas JL, Braillon A, Degott C, Quenum C. Fasting in obesity: another cause of liver injury with alcoholic hyaline? Dig Dis Sci. 1982;27:265-8.

12. Clark Jm, Brancati FL, Diehl AM. Nonalcoholic fatty liver disease. Gastroenterology. 2002; $122: 1649-57$

13. Diehl Am, Goodman Z, Ishak KG. Alcohol-like liver disease in nonalcoholics. A clinical and histologic comparison with alcohol-induced liver injury. Gastroenterology. 1988;95:1056-62.

14. Eriksson S, Eriksson KF, Bondesson L. Nonalcoholic steatohepatitis in obesity: a reversible condition. Acta Med Scand. 1986;220:83-8.

15. Grattagliano I, Vandemiale G, Caraceni P, Domenicali M, Nardo B, Cavallari A, Trevisani F, Bernardi M, Altomare E. Starvation impairs antioxidant defense in fatty livers of rats fed a choline-deficient diet. J Nutr. 2000;130:2131-6.

16. Harrison sA, Torgenson S, Hayashi P, Ward J, Schenker S. Vitamin E and vitamin $\mathrm{C}$ treatment improves fibrosis in patients with nonalcoholic steatohepatitis. Am J Gastroenterol. 2003;98:2485-90

17. Harrison SA, Neuschwander-Tetri BA. Nonalcoholic fatty liver disease and nonalcoholic steatohepatitis. Clin Liver Dis. 2004;8:861-79.

18. Ikejima K, Takei Y, Honda H, Hirose M, Yoshikawa M, Zhang YJ, Lang T, Fukuda T, Yamashina S, Kitamura T, Sato N. Leptin receptor-mediated signaling regulates hepatic fibrogenesis and remodeling of extracellular matrix in the rat. Gastroenterology. 2002;122:1399-410

19. Kakuma T, Lee Y, Higa M., Wang Z, Pan W, Shimomura I, Unger RH. Leptin, troglitazone, and the expression of sterol regulatory element binding proteins in liver and pancreatic islets. Proc Natl Acad Sci. 2000;97:8536-41

20. Koteish A, Diehl AM. Animal models of steatosis. Semin Liver Dis. 2001;21: 89-104.

21. Kugelmas M, Hill DB, Vivian B, Marsano L, McClain CJ. Cytokines and NASH a pilot study of the effects of lifestyle modification and vitamin E. Hepatology. 2003;38:413-9.

22. Laurin J, Lindor KD, Crippin JS, Gossard A, Gores GJ, Ludwig J, Rakela J, McGil DB. Ursodeoxycholic acid or clofibrate in the treatment of non-alcohol-induced steatohepatitis: a pilot study. Hepatology. 1996;23:1464-7.

23. Lavineje Je. Vitamin E treatment of nonalcoholic steatohepatitis in children: a pilot study. J Pediatr. 2000;136:734-8.

24. Leclerq Ia, Farrel GC, Field J, Bell DR, Gonzales FJ, Robertson GR. CYP2E1 and CYP4A as microsomal catalysts of lipid peroxides in murine nonalcoholic steatohepatitis. J Clin Invest. 2000;105:1067-75.

25. Lee Rg. Nonalcoholic steatohepatitis: a study of 49 patients. Hum Pathol. 1989;20:594-8

26. Lettéron P, Fromenty B, Terris B, Degott C, Pessayre D. Acute and chronic steatosis lead to in vivo lipid peroxidation in mice. J Hepatol. 1996;24:200-8

27. Lieber Cs, Leo MA, Mak KM, Xu Y, Cao Q, Ren C, Ponomarenko A, DeCarli LM Model of non-alcoholic steatohepatitis. Am J Clin Nutr. 2004;79:502-9.

28. Lin Hz, Yang SQ, Chuckaree C, Kuhajda F, Ronnet G, Diehl AM. Metformin reverses fatty liver disease in obese, leptin-deficient mice. Nat Med. 2000;6:998-1003.
29. Lindor KD, Kowdley KV, Heatchcote EJ, Harrison ME, Jorgensen R, Angulo P, Lymp JF, Burgat L, Colin P. Ursodeoxycholic acid for treatment of nonalcoholic steatohepatitis: results of a randomized trial. Hepatology. 2004;39:770-8.

30. Ludwig J, Viggiano TR, McGill DB, Oh BJ. Nonalcoholic steatohepatitis. Mayo Clinic experiences with a hitherto unnamed disease. Mayo Clin Proc. 1980;55:434-8.

31. Ludwig J, McGill DB, Lindor KD. Review: Nonalcoholic steatohepatitis. J Gastroentero Hepatol. 1997;12:398-403.

32. Matteoni CA, Younossi ZM, Gramlich T, Boparai N, Liu YC, McCullough AJ Nonalcoholic fatty liver disease: a spectrum of clinical and pathological severity. Gastroenterology. 1999;116:1413-9.

33. Marchesini G, Brizi M, Bianchi G. Metformin in non-alcoholic steatohepatitis. Lancet 2001;358:893-4.

34. Montgomery DC. Design and analysis of experiments. New York: John Wiley; 1984. $538 \mathrm{p}$

35. Neuschwander-Tetri BA, Brunt EM, Wehmeier KR, Oliver D, Bacon B. Improved nonalcoholic steatohepatitis after 48 weeks of treatment with the PPAR-y ligan rosiglitazone. Hepatology. 2003;38:1008-17.

36. Neuschwander-Tetri BA, Caldwell SH. Nonalcoholic steatohepatitis: summary of an AASLD Single Topic Conference. Hepatology. 2003;37:1202-19.

37. Okan A, Astarcioglu H, Tankurt, Sagol O, Altekin E, Astarcioglu I, Gonen O. Effect of ursodeoxycholic acid on hepatic steatosis in rats. Dig Dis Sci. 2002;47:2389-97.

38. Palmer M, Schaffener F. Effect of weight reduction on hepatic abnormalities in overweight patients. Gastroenterology. 1990;99:1408-13.

39. Pelleymounter MA, Cullen MJ, Baker MB, Hecht R, Winters D, Boone T, Collins F. Effects of the obese gene product on body weight regulation in ob/ob mice. Science. 1995;269:540-3.

40. Pessayre D, Berson A, Beco VD. Steatohepatitis-inducing drugs cause mitochondria dysfunction and lipid peroxidation in rat hepatocytes. Gastroenterology. 1998; 114:764-74.

41. Phillips MS, Liu Q, Hammond HA, Dugan V, Hey PJ, Caskey CT, Hess JF. Leptin receptor missense mutation in the fatty Zucker rat. Nat Genet. 1996;13:18-9.

42. Poulsom R. Morphological changes of organs after sucrose or fructose feeding. Prog Biochem Pharmacol. 1986;21:104-34.

43. Powell EE, Cooksley WG, Hanson R, Searle J, Halliday JW, Powell L. The natura history of nonalcoholic steatohepatitis: a follow-up study of forty-two patients for up to 21 years. Hepatology. 1990;11:74-80.

44. Ratziu V, Giral P, Charlotte F, Bruckert E, Thibault V, Theodorou I, Khalil L, Turpin G, Opolon P, Poynard T. Liver fibrosis in overweight patients. Gastroenterology. 2000;118:1117-23.

45. Rogers AE, Newberne PM. Alcoholic and nutritional fatty liver and cirrhosis. Am J Pathol. 1973;73:817-20.

46. Saviano MC, Brunetti F, Rubino A, Franzase A, Vajro P, Argenziano A, Puzziello A, Iannucci MP. Liver involvement obese children: ultrasonography and liver enzyme levels at diagnosis and during follow-up in an Italian population. Dig Dis Sci. $1997: 42 \cdot 1428-32$

47. Saxena NK, Ikeda K, Rockey DC, Friedman SL, Anania FA. Leptin in hepatic fibrosis: evidence for increased collagen production in stellate cells and lean littermates of ob/ ob mice. Hepatology. 2002;35:762-71.

48. Schaffner F, Adler M. Fatty liver hepatitis and cirrhosis in obese patients. Am J Med. 1979;67:811-6

49. Schaffner F, Thaler H. Nonalcoholic fatty liver disease. Prog Liver Dis. 1986;8 283-98.

50. Sorbi D, Boynton J, Lindor KD. The ratio of aspartate aminotransferase to alanine aminotransferase: potential value in differentiating nonalcoholic steatohepatitis from alcoholic liver disease. Am J Gastroenterol. 1999;94:1018-22.

51. Teli MR, James OFW, Burt AD, Bennett MK, Day C. The natural history of nonalcoholic fatty liver: a follow-up study. Hepatology. 1995;22:1714-9.

52. Wang RT, Koretz RL, Yee HF. Is weight reduction an effective therapy for nonalcoholic fatty liver? A systematic review. Am J Med. 2003;115:554-9.

53. Wanless IR, Lentz JS. Fatty liver hepatitis (steatohepatitis) and obesity: an autopsy study with analysis of risk factors. Hepatology. 1990;12:1106-10.

54. Weltman MD, Farrell GC, Liddle C. Increased hepatocyte CYP2E1 expression in a rat nutritional model of hepatic steatosis with inflammation. Gastroenterology. 1996;111:1645-53.

55. Younossi Z, Diehl AM, Ong JP. Nonalcoholic fatty liver disease: an agenda for clinica research. Hepatology. 2002;35:746-52.

56. Zamin IJ, Mattos AA, Zettler CG. Non-alcoholic steatohepatitis in nondiabetic obese patients. Can J Gastroenterol. 2002;16:303-8

Recebido em 25/10/2007 Aprovado em 4/6/2008 